TRABAJOS ORIGINALES

Rev. Chil. Pediatr, 69 (5); 191-194, 1998

\title{
Hernia diafragmática congénita y malformaciones asociadas
}

\author{
Enrique Daniel Austin-Ward'; Julio Nazer H. ${ }^{2}$; Silvia Castillo T. '
}

\begin{abstract}
Resumen
La hernia dialragmónca conigénita (HDC) continúa siendo une palobogia que pare en peligro la vida a pesar de los adelantas en su manejo. Las malformaciones corigénilos asociadas resultan ser uno de los foctares rás importantes que contribuyen a elevor los tasas de mortalidat en estos pocientes. En este ropajo presentamos nuesta experiencia con re ocićn a esta anomela, a cual formo parte del Estudio Colaborativc latineanericano de Malfor maciones Congenitas (ECLAMC). enocado principolme"rte en lo asociación de la HCC con atras matromaciones congénitas, y eviscmos los aspecros relacionaces con el patrón de herencia involucrado er los casos tamilicres de HDC. En este estudio enconiramos que los malformaciones mayores, especialmene las cordiacas, y un bajo peso al nacer conieren un mol pionósıç a los recién nac acs con HDC.
\end{abstract}

(Palabras clave: hernia diafromáliça congén.to, malformeciones cençérilas.'

\section{Congenital diaphragmatic hernia and associated malformations}

Congenitol diaphrogmak hern a (CDH\}) contınues to be o life-threatening condilion despite the improverrents in the managerrent. Associated congenital inalformoticns seem to be one of the most imporiant foclors that leads to on increase 'n motality ra'es in these patents. Wo present ere ow experience with this condition as part of the Lotinoomerican Coloborative Study of Congental Micltormations IECLAMC) focused on the CDH ossociation witr. ather congenitol maltermations and $c$ eview of the issues related to the pattern of inheritance nvolved in me famil.er coses of $\mathrm{CDH}$. We tound that mo or malformctions, specially cardioc and o low birth weigrt confer poor prog"csis to the newborns with, $\mathrm{CDH}$.

(Koy words: congenital diaphragmotic hernid: majer ma.forrations.l

La hernia diafragmática congénita (HDC) consiste en un defecto anatómico que resulta en una ausencia completa o parcial del diafragma ${ }^{1}$. que permite una comunicación entre las cavidades pleural y peritoneal facilitando el paso de contenido ab́dominal al tórax. Esto resulta en una compresión de las estructuras torácicas, to que lleva a un desarrollo pulmonar anormal y a un funcionamiento inadecuado del sistema cardiopulmonar desde los primeros minutos de

1. Servicio de Genética, Departamento de Medicina. Hospiral Clínico de la Universidad de Chile.

2. Unidad de Neonatología, Hospiral Clínico de la Universidad de Chile. vida extrauterina, El contenido abdominal puede herniarse a través de dos tipos de defectos diafragmáticos, de distinto origen, los cuales pueden presentarse de manera individual (lo que se conoce como agenesia parcial del diafragma) o conjunta (agenesia diafragmática bilateral o (otal).

La hernia a traves del foramen de Bochdalek se refiere al defecto de la región posterolateral izquierda del diafragma, y es causada por una falla en la fortnación o en la fusión de las membranas pleuroperitoneales ${ }^{2}$. La herniación a través del foramen de Morgagni se refiere al defecto en las regiones central y lateral derechas y es causada por una falla en el desartollo del segmento retroesternal del septum transversum. 
La HDC es una condición que pone en peligro la vida per se, pero existen varios factores que afectan la morbilidad y mortalidad de la misma, siendo uno de ellos la presencia de malformaciones congénitas asociadas. En este estudio presentamos un reporte de los casos de HDC atendidos en la Unidad de Neonatología del Hospital Clínico de la Universidad de Chile. el cual participa en el Estudio Colaborativo Latinoamericano de Malformaciones Congénitas (ECLAMC), entre el 1 de enero de 1983 y el 31 de diciembre de 1997, así como una breve revisión de los árboles genealógicos de casos familiares de HDC.

\section{Pacientes y método}

Se revisaron las fichas clínicas de todos tos pacientes con diagnóstico de hemia diafragnática congénila tanto aislada como asociada con otras malformaciones congéni. las. Se registramn Ins siguientes datos de cada paciente: sexo, peso al nacimiento, edad gestacional del recién nacido. ripo de hernia diafragrática Bochdalek, posterolateral izquierda o Morgagni. centro lateral derecha. ripo de parto. edad paterna y matema, exposición a medicamentos. historia. de enfermedad aguda o crónica en la madre, lipo de malformaciones asociactas, clasificación de las mismes se. gún fueran mayores o menores, evolución e historia relevarte de otras anomalías congénitas en otros miembros de la familia. Definimos malformación mayor como aquella que es significativa desde el punto de vista inédico, quirúr. gico o cosmetico. mientras que las mal formaciones menores son aquellas cuya corrección tiene un interés puramente cosmético?"

\section{Resultados}

En el período de 15 años antes mencionado, hubo un total de 49566 nacimientos. 3257 con malformaciones congénitas $(6,6 \%)$ mayores y menores. De estas. 32 fueron casos de HDC $(0,98 \%)$, con una prevalencia de 6,45 por 10000 nacimientos. Un caso con relajación diafragmática diagnosticada durante el procedimiento quirúrgico no fue incluido.

La relación de sexos fue de 1: 1,4 correspondiendo a 13 pacientes del sexo masculino $(40,6 \%)$ y 18 del sexo femenino $(56,3 \%)$. Un caso con síndrome de regresión caudal tenía genitales ambigutos $(3,1 \%)$. Hubo un caso de siameses toracópagos femeninos, los cuates fueron considerados como dos casos de HDC, ya que cl defecto estaba presente en ambas.
Veinticinco de los casos fueron hernias de Bochdalek $(78,1 \%), 6$ fueron hernias de Morgagni $(18,8 \%)$ y en un caso la información sobre el lado de la hernia no fue registrada $(3,1 \%)$.

Dieciséis casos fueron partos por cesárea y 15 fueron partos vaginales. El peso promedio al nacimiento fuc de $2675 \mathrm{~g}$ y la edad gestacional promedio fue de 37 semanas; 20 de los RN eran adecuados para la edad gestacional (AEG) $(62,5 \%)$; de ellos 11 murieron $(55 \%)$. Dos eran grandes para la edad gestacional (GEG) $(6,2 \%)$; ninguno de ellos murió. Además, 10 eran pequeños para la edad gestacional (PEG) (31.3\%). De estos últimos, siete murieron $y$ seis tenían otras anomalías congénitas.

Dieciséis de los 32 casos de HDC (50\%) estaban asociados con otras malformaciones (tabla I). De estos, 11 murieron $(68.8 \%)$; cinco de los fállccidos tenían sólo malformaciones cardiacas, uno solamente malformaciones del SNC y tres tenían malformaciones de ambos sistemas. De los cinco que sobrevivicron, dos tenían malformaciones menores ( $40 \%$ ), uno tenía una sola malformación mayor y los otros dos tenían dos o más malformaciones mayores. Las anomalías cardiovasculares fueron las más frecuentes con 10 casos $(62.5 \%)$, seguidas por las del SNC con $5(31,3 \%)$. Se diagnosticaron síndromes genéticos tales como el síndrome de Fryns, síndrome de regresión caudal, síndrome de

\section{Tabla 1}

Anomalías congénicas asociadas a los casos de $\mathrm{HDC}$ reportados en la Unidad de Neonatología del Hospital Clínico de la Universidad de Chile

\begin{tabular}{lc} 
Anomalías & n pacientes \\
\hline Cardiovasculares & 9 \\
SN'C & 5 \\
Defeclos de extremidades & 5 \\
Aparato genitourinario & 5 \\
Divertículo de Mecke! & 1 \\
Malrotacion intestinal & ] \\
Ano imperforado & 1 \\
Glándulas suprartenales & 2 \\
Deleclos vertebrales & ] \\
Piel & 1 \\
Bazo & 2 \\
Tracto reproductivo & ]
\end{tabular}


Roberts, pentalogía de Cantrell y sindrome de Down (tabla 2).

De los 32 casos, 18 fueron dados de alta vivos $(56,2 \%)$, tres fueron mortinatos $(9,4 \%)$ y 11 fueron morlineonatos $(34,4 \%)$. Esto representa una mortalidad de $44 \%$. De estas muertes, nueve fueron del sexo femenino $(64.3 \%)$ y cinco del sexo masculino $(35,7 \%)$.

La edad materna promedio lue de 29,4 años y la edad paterna promedio fue de $\mathbf{3 1 , 4}$ años. Las madres estuvieron expuestas a distintos medicamentos, todos ellos prescritos por sus médicos y sin efectos teratogénicos demostrados. Había historia de enfermedades crónicas en algunas madres, tales como epilepsia, artritis reumatoide, enfermedad cardíaca reumática. bocio, hipertensión e insuficiencia renal crónica, cada una de ellas en un caso; y cnfermedades agudas tales como diabetes gestacional en 2 casos y toxoplasmosis, fiebre tifoidea, infecciones del tracto urinario, choque anafiláctico, úlcera gástrica e hiperemesis gravídica, cada una de las cuales en uno de los casos.

Un paciente con hernia de Bochdalek tenía un hermano, el cual tuvo el mismo defecto.

\section{Comentario}

La HDC puede estar asociada con una o más anomalfas congénitas, ya sea que éstas aparezean como parte de un patrón desconoci. do, o bien como parte de síndromes bien identificados, algunos de ellos invalucrando anomalías numéricas y estructurales de los cromosomas y sindromes malformativos ${ }^{4}$. Nosotros encontramos una asociación con uno de estos síndromes en $18,75 \%$ de los casos (tabla 2 ).

Se observó ta clásica predominancia de las hernias de Bochdalek ${ }^{l, 2}$, ya que esta se presentó en $78 \%$ de los casos. La mortalidad del $44 \%$ registrada es algo menor que las reportadas por otros autores ${ }^{5,6}$.

Se encontraron malformaciones mayores en $34 \%$ de los pacientes. Once de ellos murieron $(79 \%)$, evidenciando que la presencia de anomalías mayores adicionales es un indicador de pobre pronóstico para los pacientes con HDC. Más detalles de la forma de presentación de las HDC se muestran en la tabla 2.

En este estudio se encontró lá misma predominancia de anomalías cardiovasculares y del sistema nervioso que la que ha sido reportada por otros autores ${ }^{7-10}$. Se encontró que la presencia de malformaciones cardíacas estuvo relacionada con un peor pronóstico, tal como fue reportado por Cunniff y cols ${ }^{11}$. Encontramos esta asociación en $80 \%$ de los pacientes con malformaciones mayores que murieron.

El bajo peso al nacimiento, que de por sî́ puede afectar la supervivencia de los RN, en pacientes con HDC contiere un peor pronostico aún, ya que encontramos $70 \%$ de mortalidad en este grupo de pacientes, en comparación con el $55 \%$ y el $0 \%$ de mortalidad registrados en los grupos de pacientes AEG y GEG respectivamentc.

No se confirmó que la incidencia de HDC estuviera influida claramente por factores como la edad materna o paterna, medicamentos y enfermedades agudas o crónicas en la madre, ni se encontraron alusiones a este respecto en reportes de otros autores.

Se ha reportado la predominancia de pacientes alectados del sexo masculino sobre el femenino en algunas series ${ }^{2 .}$ 12.14, aunque tanto nosotros como otros autores hemos encontrado lo contrario ${ }^{15}$.

\section{Tabla 2}

Formas de presentación de la HDC

\begin{tabular}{|c|c|c|c|}
\hline & $\mathbf{n}$ & $\begin{array}{l}\text { pacien- } \\
\text { tes } \%\end{array}$ & $\begin{array}{c}\% \\
\text { sobre- } \\
\text { vivencia }\end{array}$ \\
\hline Formas aisliadas & 16 & 50 & 75 \\
\hline Bochdalek & 14 & & 85,7 \\
\hline Morgagni & 2 & & 0 \\
\hline \multicolumn{4}{|l|}{ Asociacion con sindrones } \\
\hline bien identificados & 6 & 18.75 & 33 \\
\hline Trisomía 2] & $\mathbf{I}$ & & 0 \\
\hline Síndrome de Fryos & 2 & & 50 \\
\hline Peutalogía de Cantrel] & I & & 0 \\
\hline Sindrome de regresión caudal & I & & 100 \\
\hline Síndrome de Roberts* & J & & 0 \\
\hline \multicolumn{4}{|l|}{ Asociación con patrones } \\
\hline malformativos desconocidos & 10 & 31.25 & 40 \\
\hline Con anounalías menores & 2 & & 100 \\
\hline Con una anomalía mayor & 4 & & 50 \\
\hline Con dos o & & & \\
\hline más anomalias mayores & 4 & & 0 \\
\hline
\end{tabular}

* El diagnóstico definitivo de Sindrome de Roberts no fue confirmado con análisis cromosómico. 
En nuestro grupo de pacientes encontramos un caso $\mathrm{cn} \mathrm{cl}$ cual un hermano había tenido el mismo defecto. Esto ha sido reportado también por otros autores ${ }^{16-19} y$ es un hecho conocido que la HDC puede presentarse de manera esporádica como en casos familiares. Es importante determinat el tipo de herencia asociada a estos últimos por las implicaciones que tiene para el consejo genético, pero existe un gran debate con respecto al tipo de herencia de la HDC. Así, ha habido reportes de autores en los cuales se postula una forma de herencia mendeliana, en tanto que otros como Wolf ${ }^{20}$ y Norio y cols ${ }^{12}$ postulan un patrón de herencia multifactorial, micntras que un patrón de herencia recesivo es sugerido por estudios clínicos de otros autores $19,21-23$ $y$ en animales 24.25 . Crane ${ }^{26}$, Lilly y $\operatorname{cols}^{27}$ y Lipson y Williams ${ }^{28}$ han reportado pedigrís que sugieren un tipo de herencia recesiva ligada al cromosoma X. Probablemente la respuesta a este dilema provendrá en el futuro de la conjunción de hallazgos citogenéticos. estudios segrcgacionales en familias y estudios moleculares.

\section{Referencias}

I. Shandling B: The digeslive system: Peritoneum and allied structures. Diaphragınalic hernia. In: Bralow $L$. ed: Nelson Textbook of Pediarics. Phitadelptia: WB Saunders Company eds 1992; 1032-1033.

2. Giegory G, Kitferman $J$ : Lesions of the diaphragm Congenital diaphragmatic hernia. In: Rudolph A, eds.: Rudolph's Pediatrics. Londres: Prentice Hall Internacional eds 1996; 1588-1591

3. Robinsun A, Linden MG: Other isolated birth defects. In: Blackwell Scientific Publications Inc, eds. Clinical Geneties Handbook. Boston: BSP eds. 1993:522.

4. Martinez-Frías $M L$. Frias $J L$. Salvador $d$ : Clinical epidemiological analysis of malfornations. Am J Med Genel 1990 3.5: 121-125.

5. Sharland GK, Luckhart SM. Henard AJ. Allton LD: Prognosis in fetal diaphragmatic hernia. Am J Obser Gynecol 1992: 166: 9-13.

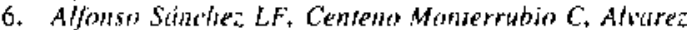
Dia: FJ, Ferre Girr M. Cotero Lavin A: Severe malformations associaled with congenital diaphragmatic hernia: conditioning factors for mortality. An Esp Pediat 1993; 39: 22-24

7. Bajaj P. Tirul A. Logemi KB. Bhem S: Congeninal diaphragmatic hernia: a retrospective autopsy study. Indian Pediatr 1991: 28: 495-500.

8. Sweed $Y$, Puri $P$ : Congenital diaphragınatio hernia: influence of associated malformations on survival. Arch Dis Child 1993; 69: 68-70.

9. Maminet-frias ML. Priet l. Urioste M. Bermejn E: Clinical/epidemiological analisys of congenital anomalies associated with diaphragmatic hernin. Ain J Med Genet 1996: 62: 101-104.

10. Russevia $R$, Koleva $V$ : Feral diaphragmatic hernia. Conconitant anonalies. Akush Ginekol (Sofia) 1997 : .36: 7.9 .

II. Cumniff $C$, Jones Kl.. Jones MC: Patterns of malformation in children with congenital diaphragmatic defects. J Pediatr 1990; 116: 2.58-261.

12. Norio R. Kauriainen H. Rapola J. Hesca R. Kekumaki $M$ : Familial congenital diaphragmatic defects: aspects of etjology. prenatal diagnosis. and treatment. Am J Med Genet 1984; 17: 471-483.

13. Torf CP, Curry CJ, Buteson TF, Honore LH: A population-based study of congenital diaphragmatic hernia. Teratology 1992; 46; 555-565.

14. Roberf $E$, Kallen $B$. Hurris J: The epidemiology of diaphragmalic hernia. Euc J Epidemiol 1997; 13; 665 673.

15. Datid TJ. IIingworh CA: Diaphragmatic hernia in the south-west of England. J Med Genet 1976: 13; 253262.

16. Passarge E, Halsey H, German J: Unilateral agenesis of the diaphragm. Human Genet 1968; 5: 226-230.

17. Ten Kate LP. Anders GJ: Unilateral agenesis of the diaphragm. Humangenetik 1970:8: $366 \cdot 367$.

18. Daent D. Passarge E: Fomiliar agenesis of the diaphragm. Birth Defects Orig Ant Ser Vul 1972: 2: 24-26.

19. Hifh DC, Carson JA, Swith El. Sarale DC. Renuert $O M$ : Familial congenital diaphragmatic hemia is an autesomal recessive variant. J Pedíatr Surg 1989: 24 $860-864$

20. Wolf $G$ : Fanilial congenital diaphragmatic defect: review and conclusions. Hum Genet 1980: 54: 1-5.

21. Furag TI. Batastakt L. Maratic M. Al-Aswidi SA. Kr.sz J: Autosomal recessive congenital diaphragmatic defects in the Arabs. Am J Med Genet 1994; 50: 30030 .

22. Mitchell SJ, Cole T, Redford DHA: Congenital diaphragmatic hernia with probable autosomal recessive inheritance in an extended consanguineous Pakistani padigree. J Med Gene1 1997; 34: 601-603.

23. Gibbs DL. Rice HE, Farrell JA, Adzick NS, Harrison $M R$ : Familial diaphragnatic agenesis: an autosomalrecessive syndrome with a poor prognosis. J Pediatr Surg 1997: 32: $366-36 \mathrm{~B}$.

24. Velentine BA. Ceroper BJ. Dietze AE. Noden DM: Canine congenital diaphrugınatic hemia. J Vet Intern Med 1988; 2: 109-112.

25. Ohkewa H. Matsimaro M. Hort T, Kashiwa $H$ : Familial congenital diaphragmatic hernia in the pigstudies on pathology and heredity. Eur J Pediatr Surg 1993: 3: 67-71.

26. Crone d: Familial congenital diagphragmatic hernia: Prenatal diagnostic approach and analysis of twelve families. Clin Genet 1979; 16: 244-2,52.

27. Lilly $J$, Malcrm P. Rosser $S$ : Anterior diaphragmatic hernia: famitial presentation. Birth Defects Orig Ant Ser X 1974: 4: 2.57-2.58.

28. Lipson $A H$, Williums $G$ : Congenital diaphragmatic hernia in half sibs. J Med Genet 1985: 22: 14,5147. 